\title{
Medellín, cincuenta años: Iglesia y signos de los tiempos Lectio inauguralis
}

\author{
Franklin Buitrago Rojas**
}

Recepción: 22 de agosto de 2018 • Aprobación: 20 de septiembre de 2018

\section{Resumen}

El Primer Congreso de Teología Latinoamericana y Caribeña se propone reflexionar sobre el documento conclusivo de la II Conferencia General del Episcopado Latinoamericano cincuenta años después de su promulgación, invitando a nuevas generaciones de teólogos a reapropiarnos del significado de este acontecimiento en el que el episcopado latinoamericana aplicó a su propio contexto la categoría signos de los tiempos propuesta por el Concilio Vaticano II: un contexto caracterizado por una conciencia creciente de las particularidades la Iglesia latinoamericana, el catolicismo popular, el desarrollo de la comunidades de base y la necesidad urgente de promoción humana y de una opción eficaz por los pobres.

Palabras clave: Conferencia de Medellín, opción por los pobres, signos de los tiempos, teología latinoamericana.

Artículo de reflexión preparado para el Primer Congreso Internacional de Teología Latinoamericana y del Caribe: 50 años de Medellín: Iglesia y signos de los tiempos, desarrollado en la Universidad Santo Tomás entre el 16 y el 19 de octubre de 2018. Citar como: Buitrago Rojas, R. (2018). Medellín, cincuenta años: Iglesia y signos de los tiempos. Lectio inauguralis. Albertus Magnus, IX(2), 73-86. Doi: https://doi.org/10.153322/5005413.5101.

** Universidad Santo Tomás, Colombia. Orcid: https://orcid.org/0000-0002-1201-6128. Correo electrónico: dec.teologia@usantotomas.edu.co 


\title{
Medellín, 50 years: Church and signs of the times Lectio inauguralis
}

\begin{abstract}
The First Congress of Latin American and Caribbean Theology intends to reflect on the conclusive document of the II General Conference of the Latin American Episcopate fifty years after its promulgation, inviting new generations of theologians to reappropriate the meaning of this event in which the Latin American episcopate applied to its own context the category Signs of the times proposed by the Second Vatican Council: a context characterized by a growing awareness of the particularities of the Latin American Church, popular Catholicism, the development of grassroots communities and the urgent need for human promotion and of an effective option for the poor.

Keywords: Conference of Medellín, option for the poor, signs of the times, Latin American theology.
\end{abstract}

\section{Medellín, cinquenta anos: Igreja e sinais dos tempos}

\section{Resumo}

O Primeiro Congresso de Teologia Latino-americana e do Caribe propõe-se refletir sobre o documento de conclusão da II Conferencia Geral do Episcopado Latino-americano cinquenta anos depois de sua promulgação, convidando as novas gerações de teólogos a reapropiar do significado deste acontecimento no qual o episcopado latino-americano aplicou no seu próprio contexto a categoria sinais dos tempos proposta pelo Concilio Vaticano II: um contexto caracterizado por uma consciência crescente das particularidades da Igreja Latino-americana, o catolicismo popular, o desenvolvimento das comunidades de base e a necessidade urgente da promoção humana e de uma opção eficaz pelos pobres.

Palavras-chave: Conferencia de Medellín, opção pelos pobres, signos dos tempos, teologia latino-americana. 


\section{Introducción}

Para la Facultad de Teología de la Universidad Santo Tomás es motivo de regocijo y de esperanza esta apertura del Primer Congreso Internacional de Teología Latinoamericana y Caribeña. Como Facultad, se ha asumido el compromiso de reflexionar sobre la realidad eclesial y social del continente americano desde una tradición propia caracterizada por la identidad dominicano-tomista y en diálogo con otros saberes y tradiciones teológicas.

Además, la Universidad ha tenido dentro de sus líneas identitarias desde su restauración en 1965 la promoción del pensamiento latinoamericano: la semana pasada se celebró en este mismo auditorio el XVII Congreso Internacional de Filosofía Latinoamericana, evento que durante más de tres décadas ha reunido a insignes pensadores de nuestro continente. Inspirados por esta tradición de nuestra facultad hermana, como Facultad de Teología nosotros emprendemos hoy un caminar con este Primer Congreso Internacional de Teología Latinoamericana y Caribeña. Nos alegra iniciar este camino en compañía de destacados teólogos de diferentes países y facultades.

\section{La importancia de la Conferencia de Medellín}

La celebración de la II Conferencia General del Episcopado Latinoamericano en la ciudad de Medellín en 1968 constituyó un acontecimiento fontal para la Iglesia católica en nuestro continente. Los numerosos congresos, coloquios, encuentros y celebraciones programados a lo largo de este año por diferentes facultades de teología, universidades católicas, conferencias episcopales y entidades eclesiales para conmemorar los cincuenta años de la II Conferencia testimonian ampliamente la importancia que el catolicismo latinoamericano y la Iglesia en general conceden a Medellín.

Por eso, evocar las grandes líneas temáticas del documento conclusivo de Medellín, sus principales intuiciones teológicas y pastorales, y recordar a las grandes figuras y protagonistas de aquel acontecimiento en el contexto de una facultad de teología, ante un auditorio de docentes y estudiantes que en su gran mayoría somos ya distantes generacionalmente de dicho acontecimiento, es una labor a la vez de reapropiación y de inspiración para nuevas generaciones. En estos días, me han preguntado varios estudiantes por qué se le da tanta importancia al Documento de Medellín mientras se pasa por alto la conmemoración de otras reuniones eclesiales o de otros documentos magisteriales. Creo que su 
pregunta es válida desde la perspectiva de nuevas generaciones de católicos y de teólogos. Espero que la reflexión y el diálogo de estos días les permita a nuestros estudiantes y a todos nosotros comprender con mayor profundidad el significado de la Conferencia de Medellín, no solo en su tiempo, sino para nuestro tiempo.

Como puede suceder con todo acontecimiento histórico que ha sido rodeado por un halo de leyenda, la Conferencia de Medellín corre el riesgo de convertirse en bandera de intereses ideológicos o de ser comprendida de manera parcial cuando desconocemos el texto y el contexto. Al inicio de este congreso, me gustaría preguntar a nuestros estudiantes cuántos hemos leído en su integridad las conclusiones de Medellín, no sea que estemos hablando de oídas. Medellín fue ante todo un acontecimiento eclesial; no es la propiedad exclusiva de un grupo o la bandera de una generación. Por eso, la invitación es doble. Por una parte, a acercarnos a este documento para los que quizá hemos oído mucho de él, pero no lo hemos leído con la atención que se merece. Y por otra, a releerlo y a comprenderlo más allá de prejuicios y cargas ideológicas que se han podido sobreponer a lo largo de las décadas. Este congreso se propone una labor de reapropiación desde el contexto mismo en el que fue escrito, desde las preguntas a las que Medellín quiso responder, y también desde el contexto nuestro, desde el contexto de quienes hoy lo leemos y desde las preguntas que nos planteamos como creyentes de nuestro tiempo.

\section{Los signos de los tiempos}

Una de las tareas de la lectio inaugural es introducir las líneas temáticas del evento, por eso, queremos compartir con ustedes algunos de los puntos de reflexión que el equipo organizador ha querido plantear para este congreso. Puntos que guiarán especialmente nuestro diálogo con los ponentes invitados y en las mesas temáticas.

Como lo dice el título mismo del Congreso, una de las categorías teológicas que hemos querido destacar dentro del Documento de Medellín (1968) es Iglesia y signos de los tiempos. En efecto, los obispos declaraban en la introducción lo siguiente:

A la luz de la fe que profesamos como creyentes, hemos realizado un esfuerzo para descubrir el plan de Dios en los "signos de nuestros tiempos". Interpretamos que las aspiraciones y clamores de América Latina son signos que revelan la orientación del plan divino operante en el amor redentor de Cristo que funda estas aspiraciones en la conciencia de una solidaridad fraternal. 
El teólogo dominico Yves Congar explica que la categoría signos de los tiempos entró en el magisterio católico gracias al impulso de Juan XXIII quien la empleó en la bula de convocatoria del Concilio Vaticano II y en la encíclica Pacem in terris (Congar, 1983, p. 238). Por su parte, el papa Pablo VI explicaba que signos de los tiempos no es una categoría teológica más, sino que ella orienta la mirada de la Iglesia hacia los acontecimientos humanos, las necesidades de los hombres, los fenómenos de la historia y las vicisitudes de nuestra vida. Ese era el tipo de mirada que los dos pontífices querían para el Concilio: una mirada que busque y descubra los signos de Dios, su presencia operante en el mundo y en la Iglesia (Pablo VI, 1964). Ese deseo de una mirada atenta a los signos de los tiempos quedaría plasmada de modo paradigmático en la constitución Gaudium et spes.

Se sabe que una de las claves para comprender la Conferencia de Medellín es recordar que fue ella convocada con el objetivo de aplicar las disposiciones del Concilio Vaticano II a la realidad de la Iglesia en América Latina. Por eso, la mirada atenta a los signos de los tiempos va a configurar de modo eminente la reflexión de la II Conferencia General del Episcopado Latinoamericano. En una obra dedicada a los signos de los tiempos, el profesor Patricio Merino Beas, quien fuera por varios años docente y decano de nuestra facultad, explica que toda la estructura del Documento de Medellín está basada en la metodología que se desprende de la categoría signos de los tiempos (2014, p. 84). De hecho, dos de las siete ponencias que se hicieron durante la Conferencia de Medellín estuvieron dedicadas explícitamente a los signos de los tiempos: una del obispo panameño Marcos McGrath (2018) "Los signos de los tiempos en América Latina hoy" y otra del secretario mismo de la Conferencia de Medellín, monseñor Eduardo Pironio (2018), titulada "Interpretación cristiana de los signos de los tiempos hoy en América Latina". Tres son los grandes signos de los tiempos según la interpretación de McGrath: a) el cambio que afecta a todas las dimensiones de la vida humana; b) la valoración de lo temporal, una visión positiva de la creación y del desarrollo; y c) el enfoque mundial que señala la interdependencia y la necesaria integración de las naciones en América Latina. Al releer el Documento de Medellín desde la categoría signos de los tiempos, queremos preguntarnos en este congreso ¿cuáles son los signos de los tiempos en la América Latina y el Caribe hoy cincuenta años después? ¿Son los mismos? ¿Qué signos nuevos han ido apareciendo en el horizonte de lo social y de lo eclesial?

Al mismo tiempo, una relectura contemporánea del Concilio Vaticano II o de las conclusiones de la Conferencia de Medellín no puede pasar por alto que diversas voces han hecho notar un uso a veces ambiguo o poco claro de la categoría signos de los tiempos. Teólogos como Marie-Dominique Chenu se dedicaron 
por ello en los años posteriores al Concilio a una clarificación y profundización teológica de dicha categoría. Signos de los tiempos es usado a veces para designar los anhelos de humanización, de liberación y de progreso de los pueblos y de los individuos en un momento concreto de la historia; en otras ocasiones, se usa para designar lo que Dios mismo está haciendo en medio del mundo, los signos de su presencia. Una identificación demasiado rápida de estos dos significados de la categoría signos de los tiempos, uno más sociológico y el otro más teológico, fue el origen de un cierto número de tensiones en el posconcilio.

Por eso, otras conferencias del Consejo Episcopal Latinoamericano (Celam) posteriores a Medellín han buscado profundizar en el sentido cristológico o pneumatológico a esta categoría. Con la distancia del tiempo, un lector contemporáneo puede percibir un cierto optimismo demasiado amplio, quizá propia de la década de 1960, cuando se veía como inminente la llegada de una nueva era de desarrollo y de progreso para los pueblos de América Latina o el despuntar de una primavera eclesial. Las décadas sucesivas al Vaticano II y a Medellín fueron también décadas de luchas sociales infructíferas, de revoluciones sociales, culturales y militares fallidas, para muchos fueron también tiempos de invierno eclesial. ¿Cómo renovar y actualizar esa apuesta por una mirada que descubra los signos de la presencia de Dios en nuestro mundo?

Al resaltar la categoría signos de los tiempos en el título de este congreso, quisimos mostrar que la interpretación de dichos signos sigue siendo una tarea para cada generación de cristianos, una tarea a la que Cristo mismo urge a sus discípulos, la tarea que el Concilio Vaticano II y la Conferencia de Medellín asumieron en su momento y contexto histórico, y una tarea para nosotros hoy. Pero una tarea que no se puede tomar a la ligera sino que debe ser asumida en toda su complejidad. Pensar teológicamente desde América Latina y el Caribe implica identificar, comprender y discernir esos signos de los tiempos, antiguos y nuevos, en los que los anhelos de humanización y de liberación se encuentran con la voluntad salvífica de Dios.

\section{Iglesia y teologías latinoamericanas}

En el título de este congreso, se unen dos categorías: Iglesia y signos de los tiempos. Recordemos que la Iglesia misma es un signo de los tiempos y lo es en el doble sentido de la expresión. Ella, como realidad teológica e histórica, es un signo visible de la voluntad salvífica de Dios, es resultado visible de la acción redentora de Cristo en medio de los hombres. La Iglesia de cada tiempo es también 
lugar donde se expresan las esperanzas de salvación de los hombres en medio de las vicisitudes de la historia. En el Documento de Medellín, se expresa esta doble dimensión de la Iglesia signo de los tiempos: una Iglesia que toma en cuenta su realidad teológica y su realidad histórica que implica dimensiones sociales y culturales. No olvidemos que cada uno de nosotros aquí presentes representa y encarna rostros de esa misma Iglesia: mujeres, hombres, laicos, religiosos, ministros ordenados, casados, célibes y fieles de diferentes confesiones. Es desde la actualidad de nuestra doble pertenencia a la Iglesia y al mundo con todas sus complejidades, sociales, culturales, políticas y económicas que osamos dirigir la mirada para escrutar, discernir e interpretar los signos de nuestro tiempo. Creemos que la Iglesia puede descubrir también en ella misma los signos de los tiempos, dialogar sin oposición al mundo y sin diluirse en él.

Otra de las líneas temáticas de nuestro congreso es Medellín y teologías latinoamericanas. Muchos analistas e historiadores de la II Conferencia General del Episcopado Latinoamericano destacan que Medellín fue un momento de toma de conciencia de la Iglesia latinoamericana, una etapa fundamental en el forjar una autoconciencia. La gran mayoría de los obispos que participaron en la Conferencia lo habían hecho unos años antes en el Concilio Vaticano II. Eran conscientes de que sus iglesias tenían problemas particulares y que las grandes líneas temáticas del Concilio todavía seguían orientadas principalmente por las preocupaciones del episcopado europeo, como el tema de la Iglesia frente al mundo moderno. Pero América Latina tenía sus problemas, sus preocupaciones y sus realidades específicas que no iban a ser reflexionadas por teólogos de otros continentes. Recuerdo una lectio magistral dada por Gustavo Gutiérrez hace unos años cuando la orden de los dominicos le confirió el grado de Maestro en Sagrada Teología. Fray Gustavo, recordando su participación en la última sesión del Vaticano II, rememoraba que al final del Concilio entre muchos episcopados, especialmente latinoamericanos y africanos, quedó la idea de que al Vaticano II le quedó faltando un documento específico en el que se tratara un tema que aquejaba a millones de católicos: la pobreza. En cierto modo, Populorum progressio de Pablo VI fue una respuesta a esa inquietud. Medellín fue una respuesta a esa inquietud. Esta autoconciencia de la Iglesia latinoamericana, de sus realidades y desafíos propios, explica en gran medida la orientación y el tono del Documento de Medellín.

En su mensaje a los pueblos de América Latina al finalizar la Conferencia de Medellín, los obispos declaraban: "Como hombres latinoamericanos, compartimos la historia de nuestro pueblo. El pasado nos configura definitivamente como seres latinoamericanos; el presente nos pone en una coyuntura decisiva y el futuro nos exige una tarea creadora en el proceso de desarrollo" (II Conferencia 
General del Episcopado Latinoamericano, 1968). Y más adelante en la introducción a las conclusiones, afirman los obispos: “Tomamos conciencia de la 'vocación original' de América Latina: vocación a aunar en una síntesis nueva y genial, lo antiguo y lo moderno, lo espiritual y lo temporal, lo que otros nos entregan a nuestra propia originalidad" (n. 7).

La toma de conciencia de la particularidad latinoamericana por un buen número de obispos de nuestro continente se percibe también en la dinámica que toma el Celam como órgano de colegialidad y de liderazgo frente a las prioridades, las apuestas y los proyectos lanzados por la Conferencia de Medellín. El episcopado latinoamericano a partir de Medellín fue pionero en aplicar las ideas de colegialidad impulsadas por el Concilio Vaticano II. Todos estos elementos hacen de Medellín un momento histórico dentro del caminar de la Iglesia latinoamericana y un punto de referencia dentro de la evolución de la Iglesia católica en la segunda mitad del siglo XX.

La toma de conciencia de lo local y de lo regional manifestada por los obispos en la Conferencia corresponde también con un despertar de la conciencia de los teólogos latinoamericanos. Medellín fue también obra de una generación de teólogos que decidieron dejar de verse a sí mismos como teólogos de periferia que replicaban lo pensado en el centro de la cristiandad, para comprender que tenían una originalidad de aportar a la teología y a la vida eclesial. Quizá por primera vez los teólogos de nuestro continente le apostaron a "beber en su propio pozo", para retomar la conocida expresión de Gustavo Gutiérrez. Para nuestro continente, Medellín marcó un punto de inflexión en el método teológico que ya se había anticipado de modo paradigmático en la constitución Gaudium et spes del Vaticano II en que se parte del análisis de la realidad con su complejidad humana para iluminarla a la luz de Cristo y para proponer un camino de acción pastoral. Hablamos hoy de teología india, teología negra, teologías de género, teologías campesinas, teología de la migración, teología del pueblo, sin olvidar, por supuesto, las teologías de la liberación. La línea temática Medellín y teologías latinoamericanas, dentro de este congreso, quiere profundizar en este giro en la manera de hacer teología que ha sido particularmente fecundo en nuestro continente.

Dos mesas temáticas dentro de nuestro congreso se ocuparán del lugar que tuvo dentro de la Conferencia de Medellín la promoción humana y la opción por los pobres. Como ya se señaló, los obispos participantes en la II Conferencia tenían conciencia de las particularidades del contexto de sus iglesias latinoamericanas en el que las realidades de pobreza, injustica, exclusión y violencia constituían y constituyen una prioridad. Los obispos afirman de manera decidida en Medellín: “Como Pastores, con una responsabilidad común, queremos comprometernos 
con la vida de todos nuestros pueblos en la búsqueda angustiosa de soluciones adecuadas para sus múltiples problemas. Nuestra misión es contribuir a la promoción integral del hombre y de las comunidades del continente" (II Conferencia General del Episcopado Latinoamericano, 1968). La primera área sobre la que recae su solicitud pastoral es "la promoción del hombre y de los pueblos hacia los valores de la justicia, la paz, la educación y la familia".

Recordemos que la encíclica reciente Populorum progressio, así como el discurso de apertura de la conferencia por parte del mismo Pablo VI, los animaba en esa misma vía:

Nuestro primer deber en este campo es afirmar los principios, observar y señalar las necesidades, declarar los valores primordiales, apoyar los programas sociales y técnicos verdaderamente útiles y marcados con el sello de la justicia, en su camino hacia un orden nuevo y hacia el bien común, formar sacerdotes y seglares en el conocimiento de los problemas sociales, encauzar seglares bien preparados a la gran obra de los mismos, considerándolo todo bajo la luz cristiana que nos hace descubrir al hombre en el puesto primero y los demás bienes subordinados a su promoción total en el tiempo y a su salvación en la eternidad.

Aparece entonces con toda claridad en la conciencia eclesial que la evangelización no puede considerarse separada de la promoción humana y que en un contexto como el latinoamericano esa promoción implica una opción clara por los más desfavorecidos dentro la sociedad. La opción por los pobres, el compromiso por la justicia y la promoción humana no son tareas o compromisos de un sector de la teología o de movimientos específicos dentro de la Iglesia: son una opción y un compromiso de todo bautizado, de todo teólogo. Es algo que nunca deberíamos perder de vista. En ese mismo contexto, Medellín habla de una Iglesia pobre que sea capaz de reconocer a Dios en el pobre. Un programa de vida que nos sigue interpelando.

Los que han tenido la oportunidad de releer el Documento de Medellín a cincuenta años de distancia estarán de acuerdo conmigo en que una de las cosas que más llaman la atención es corroborar la actualidad del diagnóstico de realidad que hicieron los obispos hace cinco décadas. Los obispos describen con estas palabras las condiciones de vida "menos humanas" en nuestro continente:

La miseria que margina a grandes grupos humanos como hecho colectivo es una injusticia que clama al cielo. Quizás no se ha dicho suficientemente que los esfuerzos llevados a cabo no han sido capaces, en general, de asegurar el respeto y la realización de la justicia en todos 
los sectores de las respectivas comunidades nacionales. Las familias no encuentran muchas veces posibilidades concretas de educación para sus hijos. La juventud reclama su derecho a ingresar en la universidad o centros superiores de perfeccionamiento intelectual o técnico-profesional; la mujer, su igualdad de derecho y de hecho con el hombre; los campesinos, mejores condiciones de vida; o si son productores, mejores precios y seguridad en la comercialización.

La creciente clase media se siente afectada por la falta de expectativa. Se ha iniciado un éxodo de profesionales y técnicos a países más desarrollados. Los pequeños artesanos e industriales son presionados por intereses mayores y no pocos grandes industriales de Latinoamérica van pasando progresivamente a depender de empresas mundiales. No podemos ignorar el fenómeno de esta casi universal frustración de legítimas aspiraciones que crea el clima de angustia colectiva que ya estamos viviendo. La falta de integración socio-cultural, en la mayoría de nuestros países, ha dado origen a la superposición de culturas. En lo económico se implantaron sistemas que contemplan solo las posibilidades de sectores con alto poder adquisitivo. Esta falta de adaptación a la idiosincrasia y a las posibilidades de nuestra población, origina, a su vez, una frecuente inestabilidad política y la consolidación de instituciones puramente formales. A todos ellos debe agregarse la falta de solidaridad, que lleva, en el plano individual y social, a cometer verdaderos pecados, cuya cristalización aparece evidente en las estructuras injustas que caracterizan la situación de América Latina. (II Conferencia General del Episcopado Latinoamericano, 1968, n. 1)

Constatar la actualidad de este diagnóstico hecho por los obispos en Medellín nos confirma la profundidad de su análisis y su clarividencia profética, pero también nos deja un gran sinsabor. El sinsabor de preguntarnos por qué cincuenta años después realidades como la pobreza, la exclusión y la falta de oportunidades siguen existiendo y siguen siendo mayoritarias entre la población latinoamericana.

Cualquier lector del Documento de Medellín puede preguntarse por qué, a pesar del compromiso valiente y del tono programático de los obispos en 1968, esas realidades siguen ahí y parecen aumentar. Si mirásemos Medellín como un plan de acción de una organización, no podemos evitar pensar en un fracaso o en una tardanza extraordinaria en ver los resultados. ¿Por qué tantos puntos del 
programa de Medellín siguen pendientes? Creo que esa es una pregunta que debería acompañarnos durante estos días de Congreso. Es una pregunta a la que se le han dado numerosas respuestas: se habla de la resistencia de sectores influyentes de la jerarquía eclesiástica en Latinoamérica y en la curia romana motivados por la comodidad de una pastoral de conservación o por una alianza con el poder político que se veía perjudicado por el tono de reforma social de Medellín; alianzas especialmente con dictaduras militares o sectores oligárquicos conservadores que temían el avance del socialismo comunista en el continente.

Se habla de un retroceso o involución en conferencias posteriores que habrían vuelto sobre esquemas eclesiológicos y pastorales preconciliares. Otros hablan de una cierta ingenuidad en la lectura histórica de los procesos latinoamericanos fomentada por un desarrollismo que creía en la transformación estructural del continente como algo inminente y que era deber de la Iglesia sumarse a esa transformación dando su mejor contribución. Algunos hablarán de la falta de compromiso político de las generaciones más recientes o del tono individualista y espiritualizante de los nuevos movimientos eclesiales. Otros dirán que la evolución religiosa del continente simplemente siguió otras sendas. Como decía alguien con ironía: la Iglesia en América Latina optó por los pobres, pero los pobres optaron por el pentecostalismo, más interesado en una liberación basada en la prosperidad individual. ¿Qué queda de la opción por los pobres en un continente donde la pobreza sigue siendo una realidad cruel y mayoritaria? Es una de las preguntas que acompañarán a esas dos mesas temáticas.

Otra línea temática que quisiéramos abordar dentro de este congreso tiene que ver con Medellín y con los modelos eclesiológicos. El Documento de Medellín da un impulso decidido a comunidades de base, tema al que se dedica toda una sección del documento y que se destaca como una de sus principales apuestas. Sin embargo, el número y la vitalidad de las comunidades de base en América Latina hoy no son lo que soñaron los obispos en Medellín. En su lugar, el mundo católico está configurado de manera creciente por nuevos movimientos eclesiales que, junto con las parroquias, son los principales lugares de pertenencia eclesial, de formación y de vivencia de la fe. Incluso quienes culpan al clero de ser responsable del estancamiento de las comunidades de base pueden ver que los movimientos eclesiales parecen ser más sólidos ante dificultades semejantes ¿Qué pasó con las comunidades de base y sobre todo con la eclesiología que estaba a la base de dichas comunidades?

Como miembro de una comunidad religiosa, me permito lanzar también una pregunta sobre la visión y el proyecto que Medellín tuvo para la vida religiosa. Muchas comunidades religiosas, sobre todo femeninas, apostaron a fondo 
por las opciones de Medellín, por el aggiornamento, como se decía en ese entonces: inserción en medios sociales difíciles, transformación de obras y de comprensión del propio carisma, abandono de estructuras anticuadas y de observancias regulares para acercarse al pueblo. Tras décadas de una labor heroica y de generaciones numerosas de religiosos comprometidos en esta línea, constatamos un declive numérico de religiosos y de obras apostólicas.

No puedo evitar hacer un paralelo con el declive de las comunidades eclesiales de base frente al crecimiento de nuevos movimientos eclesiales: también la vida religiosa ha visto el nacimiento de nuevas comunidades o la llegada de nuevas vocaciones cuyas aspiraciones se parecen más a lo que la generación de Medellín llamaría una vida religiosa tradicional, antigua y caduca. ¿Qué ha pasado en el panorama religioso de América Latina? ¿Qué cambios de sensibilidad, de orientaciones y de opciones vemos en nuestros contextos eclesiales? Creo que ante estos fenómenos, que tal vez no se preveían en 1968, deberíamos tener una mirada atenta para discernir los signos de los tiempos, para escuchar los anhelos de liberación de nuestros contemporáneos y las manifestaciones de la voluntad salvadora de Dios.

Por último, una de nuestras mesas temáticas estará dedicada a la evangelización y al crecimiento en la fe. Otra opción del Documento de Medellín, que sigue siendo actual y vigente, es la necesidad de una conversión pastoral de la Iglesia: ella implica una necesaria renovación de las estructuras eclesiales y de las acciones evangelizadoras. Una tarea urgente y amplia que, al parecer, sigue pendiente, como nos lo recuerdan el Documento de Aparecida y el magisterio del papa Francisco. Medellín tiene la característica de ser un documento programático, cada sección concluye con orientaciones pastorales en las que se nombran responsables de acciones y prioridades. Medellín apostó por la educación, la catequesis, las juventudes, las familias, el diálogo, la cooperación, la pastoral de conjunto, la pastoral social y los medios de comunicación: ve la Iglesia como un gran conjunto orgánico en movimiento y en acción, al servicio del desarrollo material y espiritual de nuestros pueblos.

\section{Conclusión}

Ese impulso evangelizador, esa generosidad y ese coraje del futuro deberían contagiarnos durante estos días. Celebramos cincuenta años, no solo de un documento, sino de un caminar eclesial: un caminar con luces y sombras, un caminar de discipulado y de misión, y un caminar de compromiso evangélico que nos han 
dejado testimonios tan grandes como el ministerio de monseñor Oscar Arnulfo Romero canonizado, junto a Pablo VI, otro de los grandes impulsores de la Conferencia de Medellín. Que estos días de diálogo nos animen en nuestro compromiso de bautizados, de teólogos, de docentes y estudiantes, reconociendo que las voces proféticas de nuestro episcopado hace cincuenta años siguen resonando como anhelos de liberación, como sed de Dios, en muchos de nuestros hermanos de América Latina y el Caribe.

\section{Referencias}

II Conferencia General del Episcopado Latinoamericano. (1968). Documento de Medellín.

V Conferencia General del Episcopado Latinoamericano. (2007). Documento de Aparecida.

Azcuy, V. R., Schickendantz, C. F. y Silva Arévalo, E. A. (Eds.) (2013). Teología de los signos de los tiempos latinoamericanos: horizontes, criterios y métodos. Santiago de Chile, Chile: Universidad Alberto Hurtado. DOI: https:/doi. org/10.1177/0040563914548659h

Chenu, M.-D. (1968). Los signos de los tiempos. En Los cristianos y la acción temporal (pp. 35-54). Barcelona, España: Estela.

Chenu, M.-D. (1970). Los signos de los tiempos: reflexión teológica. En Y. Congar (Ed.), La Iglesia y el mundo de hoy (pp. 253-278). Madrid, España: Taurus.

Congar, Y. (1983). El Espíritu Santo. Barcelona, España: Herder.

Fisichella, R. (1989). Los signos de los tiempos en el contexto contemporáneo. Medellin, 65, 55-71.

Gutiérrez, G. (2001). Situación y tareas de la teología de la liberación. Selecciones de Teología, 157, 3-13.

Jaramillo, J. (1992). Los efectos de Medellín en la pastoral latinoamericana de 1968 a 1990. Medellín, 71, 487-507.

McGrath, M. (2018). Los signos de los tiempos en América Latina hoy. Medellín, 171, 51-78.

Merino Beas, P. (2014). La categoría teológica signos de los tiempos: desde el Concilio Vaticano II al Pentecostés de Aparecida y Francisco. Bogotá, Colombia: Universidad Santo Tomás.

Pablo VI. (1964). Ecclesiam suam. Ciudad del Vaticano: Editrice Vaticana.

Pablo VI. (1965). Gaudium et spes. Ciudad del Vaticano: Editrice Vaticana.

Pablo VI. (1967). Populorum progressio. Ciudad del Vaticano: Editrice Vaticana. 
Pironio, E. (2018). Interpretación cristiana de los signos de los tiempos hoy en América Latina. Medellín, 171, 79-98.

Saranyana, J. I. (Dir.) (2002). Teología en América Latina. Vol. III: El siglo de las teologías latinoamericanas 1899-2001. Madrid, España: Iberoamericana.

Secretariado General del Celam. (1977). Medellín, reflexiones en el Celam. Madrid, España: Biblioteca de Autores Cristianos. 